\title{
The Relationship between Executive Function and Falls and Gait Abnormalities in Older Adults: A Systematic Review
}

\author{
Fiona C. Kearney ${ }^{a}$ Rowan H. Harwood ${ }^{a}$ b John R.F. Gladman ${ }^{a}$ \\ Nadina Lincoln ${ }^{a}$ Tahir Masud ${ }^{a, b}$ \\ a University of Nottingham, and bottingham University Hospitals, NHS Trust, Nottingham, UK
}

\section{Key Words}

Older adults · Executive function · Falls · Gait

\begin{abstract}
Background/Objectives: Older adults with dementia have at least a twofold increased risk of falls. Multi-factorial interventions have failed to demonstrate a reduction in falls in this group. Improved understanding of specific cognitive factors and their relationship to gait, balance and falls is required. Methods: Systematic searches of Medline, Embase, PsycInfo, and CINAHL databases from inception to April 2011 were conducted to identify prospective studies in older adults examining executive function and its relationship with falls, balance and gait abnormalities. Two independent reviewers extracted data on study populations, executive function measures and study outcomes. Results: Of 8,985 abstracts identified, 14 studies met inclusion criteria. Eleven studies examined executive function and falls. The remaining studies examined executive function and gait speed decline. Nine studies examining executive function and falls found a relationship between poor executive function and increased fall risk. All 3 studies examining executive function and gait found an association between poor executive function and declines in gait speed. Impaired executive function was associated with more serious falling patterns. Conclusions: Executive function was associated with falls and gait speed slowing in older adults. Future research should consider executive dysfunction as a training target for fall prevention, or as a factor mediating the failure of conventional fall prevention interventions.


Kearney et al.: The Relationship between Executive Function and Falls and Gait Abnormalities in Older Adults: A Systematic Review

\section{Introduction}

Falls are an important cause of morbidity and mortality in older adults with dementia. Older adults with cognitive impairment have at least a twofold increased risk of falls compared with cognitively intact older adults [1], equating to an annual fall incidence of $60-80 \%$ in this population [2,3]. People with cognitive impairment who fall are significantly more likely to sustain serious injuries and require admission to long-term care facilities [4, 5].

There has been extensive research into falls and fall prevention in older people with multifactorial interventions reducing fall risk in this group [6]. However, managing falls in people with dementia has proven difficult, and multifactorial interventions fail to demonstrate the same reduction $[7,8]$. Potential reasons include more severe gait and balance impairments in this group [9], unresponsiveness to fall prevention programmes, or the possibility of dementia-related cognitive factors contributing to fall risk.

In a seminal paper in 1997, Lundin-Olsson et al. [10] demonstrated that those who 'stopped walking when talking' were more likely to fall, indicating that gait was attentionally demanding. This prompted extensive investigation of 'dual-task deficits' in gait (declines in gait performance during simultaneous performance of a secondary cognitive task), as a predictor of falls [11] and led to interest in other cognitive domains, such as executive function, as potential contributors to fall risk in dementia.

There is no generally accepted definition of executive function, but there is consensus that executive function consists of those functions involved in decision-making [12]. This includes the abilities to reason and solve problems, initiate and maintain tasks, cognitive flexibility to adapt to changing contingencies, and it incorporates attention, working memory, and abstract reasoning [13]. Early studies in this area demonstrated that impaired executive function was associated with an increased risk of injurious falls in older adults and also explained the variance in fall risk in an inpatient rehabilitation population spanning all age groups $[14,15]$. More recently a decline in executive function was associated with an increased risk of falls in older adults with Alzheimer's disease [16], but also in older adults without overt cognitive impairment [17] suggesting that cognitive risk factors for falls in dementia may evolve before dementia is clinically evident.

Measures of gait, such as stride length and swing time variability, predict falls in a community-dwelling cohort of older adults [18]. Several studies support the association of gait and balance abnormalities in older adults with dementia [19-21]. A causal relationship between impaired executive function and gait changes remains to be proven, but ample evidence exists to demonstrate an association between the two [22-26]. This work suggests that executive function and gait impairments may underpin the increased risk of falls in dementia. However, tests of executive function are not part of routine fall risk assessment.

No systematic review has evaluated prospective studies examining the association of executive function and falls or changes in balance and gait parameters to determine if tests of executive function are predictive of future fall risk or gait impairment. We hypothesised that impaired executive function in older adults would be associated with a higher subsequent fall risk. We also hypothesised that impaired executive function would be associated with changes in gait or balance parameters that are known to increase fall risk.

\section{Methods}

A systematic review of existing literature was performed, following the MOOSE (meta-analysis of observational studies in epidemiology) guidelines and the PRISMA statement [27, 28]. Eligible prospective studies included older adults with or without dementia and examined the association between a specified measure of executive function and falls or changes in gait or balance. Studies solely demonstrating the relationship 
Kearney et al.: The Relationship between Executive Function and Falls and Gait

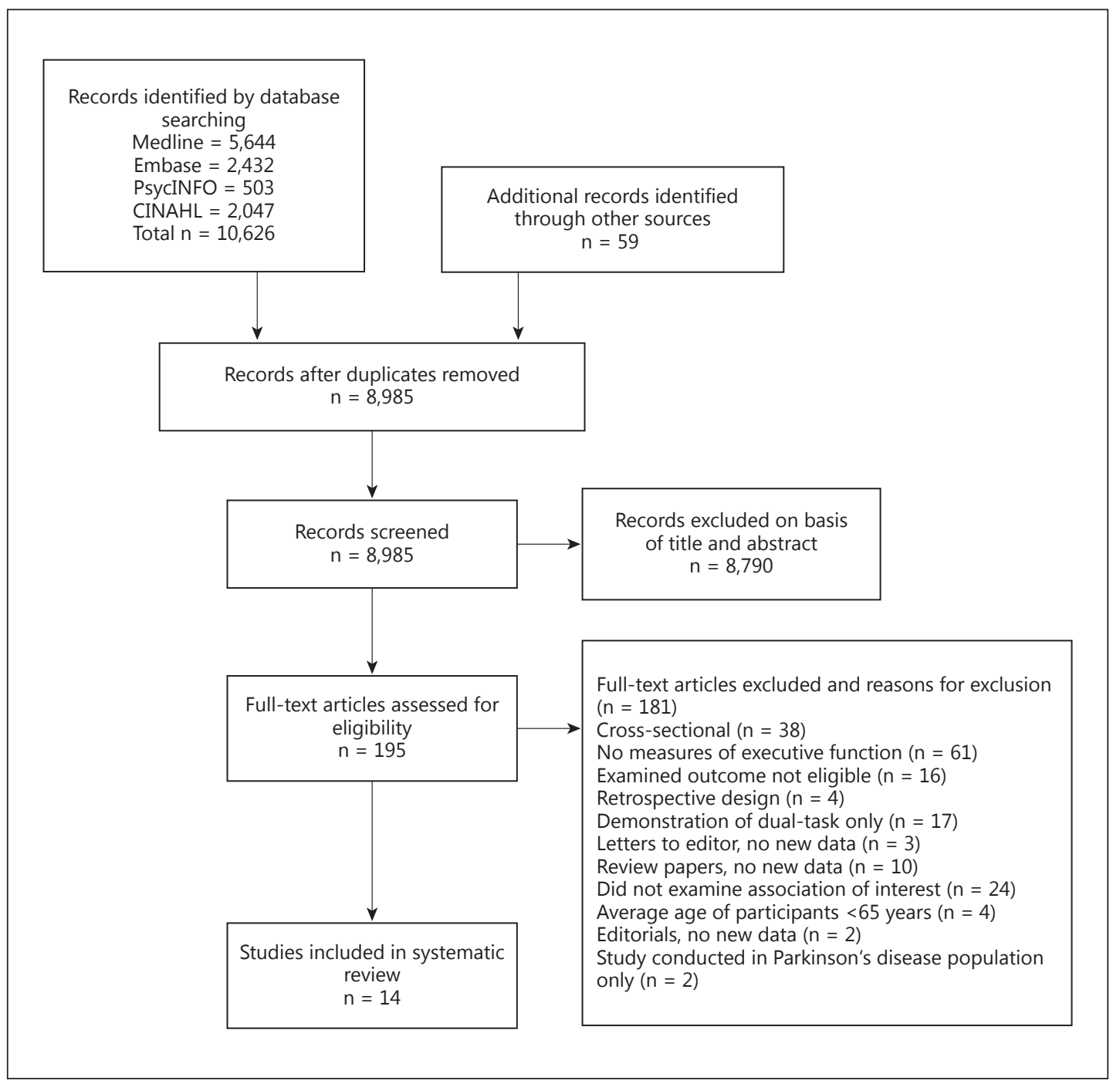

Fig. 1. Flow diagram for identification of included studies (adapted from the PRISMA statement [27]).

between dual-task decrements and incident falls have been extensively reported elsewhere and were excluded from this review [11]. Due to lack of consensus definition of executive function, for the purposes of this review, measures of executive function in its broadest definition, incorporating attention and working memory, and deemed appropriate in the textbook Neuropsychological Assessment [12], were included. Participants in eligible studies had to be over 65 years old, but studies with mean ages and standard deviations indicating the majority of participants were over 65 years were also eligible for inclusion. Studies reporting serious or injurious falls as outcome measures were considered eligible for inclusion as they have been used as a proxy measure for fall reporting in dementia populations. For inclusion, studies must have examined an association between measures of executive function and one of the stated outcomes. If this association was not reported in the research study, nor available from the authors, the study was excluded.

One author (F.K.) searched the electronic databases Medline, Embase, PsycINFO, and CINAHL from inception to April 2011 using free text search terms. Figure 1 summarises the study identification process. Reference lists of key studies and review articles known to the authors were also reviewed to identify potential papers not identified by the search criteria. The results from each database search and records identified through other sources were combined and duplicate abstracts removed. The remaining abstracts were screened to identify full-text articles for independent assessment of eligibility by one author (F.K.) and 
either of two other authors (R.H. or T.M.). Disagreements about inclusion were resolved by discussion and further assessment by the third author who had not already screened the abstract (R.H. or T.M.). Data was extracted from the papers using a standardised form and is presented in tables 1-3. The quality of each study was assessed using a modified version of the Newcastle-Ottawa quality assessment scale, but no study was excluded on the basis of this scale [29]. Meta-analyses were planned if there was adequate uniformity in testing parameters and outcome measures.

\section{Results}

The initial search yielded 8,985 abstracts, from which 195 full-text articles were chosen for further assessment, in turn, identifying 14 studies for inclusion [14, 17, 30-41]. Due to heterogeneity of baseline measures, analysis methods and follow-up periods, meta-analyses of studies were not feasible. Therefore, relevant findings, interpretation, and implications for clinical practice and future research are presented in narrative form. The systematic search identified 10 review articles; however, none of these articles systematically appraised prospective cohort studies examining the association between executive function and falls or gait and balance outcome measures.

Tables 1-3 summarise the 14 included studies. Six studies used varying scoring methods of the Trail Making Test A (TMT-A) or B (TMT-B) as a stand-alone measure of executive function $[14,17,30-33]$ and are discussed together (table 1 , whereas table 4 provides a description of cognitive tests). These 6 studies assessed a total of 1,768 community-dwelling older adults and all recorded falls as a primary outcome measure. Five studies excluded participants with cognitive impairment determined by the Mini Mental State Examination $[17,30,31,33]$. Two studies included participants $<65$ years old $[14,32]$, but the mean age of participants was greater than 70 years in each study. Five studies reported an association between poor TMT performance and falls $[14,17,30,32,33]$ with 4 studies examining the association between TMT and recurrent or injurious falls [14, 17, 30,32]. Only 1 study $(\mathrm{n}=$ 216) found no association between TMT measure and fall status [31]. This study reported recurrent falls in 55 participants, but subgroup analysis was not performed to examine if an association between executive function and recurrent falls existed.

One study reported a twofold increased risk of recurrent or injurious falls in those with poorer cognitive flexibility as measured by $\triangle$ TMT (time to complete TMT-B - time to complete TMT-A) [30]. Using classification and regression tree analysis, the authors also reported that in participants at a high risk of falls on the basis of physiological assessment, impaired executive function was the next discriminating risk factor for injurious or recurrent falls [30]. Another study reported that in a subgroup that had never fallen at baseline normalised TMT-B scores were significantly worse in recurrent fallers compared to non-fallers (odds ratio, OR, 1.82, 95\% confidence interval, CI, 1.1-3.0) [17]. They also found that a composite executive function score (Mindstreams ${ }^{\circledR}$ ) predicted future falls with participants in the worst executive function quartile having a threefold increased risk of falls and an earlier transition from nonfaller to faller [17]. In a further study, those with slower performances on TMT-B (>180 s) were almost twice as likely to sustain an injurious fall (OR 1.9, 95\% CI 1.1-3.2) [14].

Pijnappels et al. [32] found significantly poorer performance on TMT-B in recurrent fallers, compared to non-fallers ( 94 vs. 66 s, p < 0.001). Further evaluation using path analysis indicated that slow cognitive spatial processing indirectly increased fall risk by influencing reaction time and balance. Finally, slower performance on the Trail Walking Test, an adaptation of the TMT, was significantly associated with future falls (78 $\mathrm{s}$ in fallers vs. $62 \mathrm{~s}$ in non-fallers) with a weaker association found between TMT-A and falls in the same group [33]. 
Kearney et al.: The Relationship between Executive Function and Falls and Gait Abnormalities in Older Adults: A Systematic Review

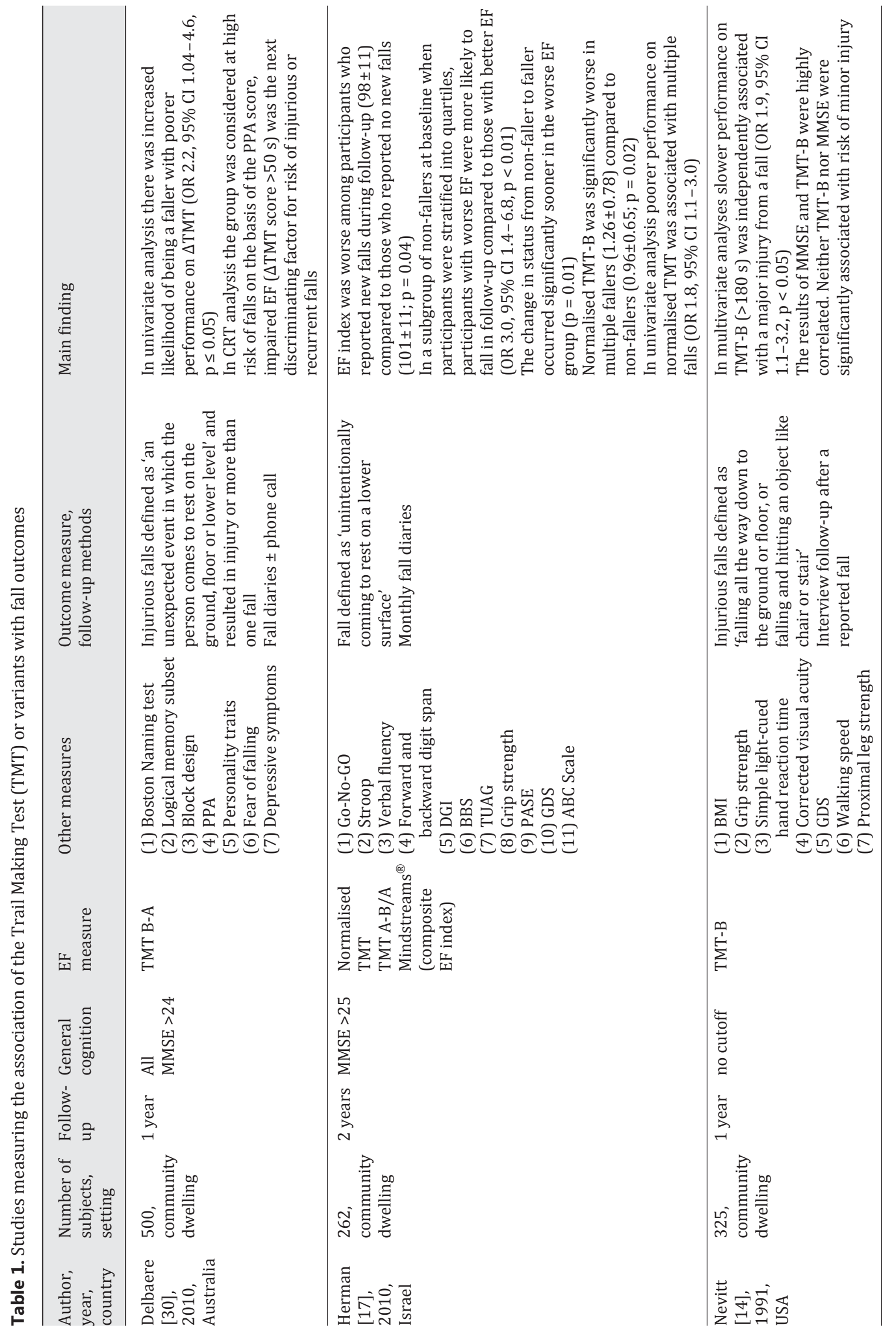


Dementia

Cognitive Disorders
Dement Geriatr Cogn Disord 2013;36:20-35

DOI: 10.1159/000350031

Kearney et al.: The Relationship between Executive Function and Falls and Gait Abnormalities in Older Adults: A Systematic Review
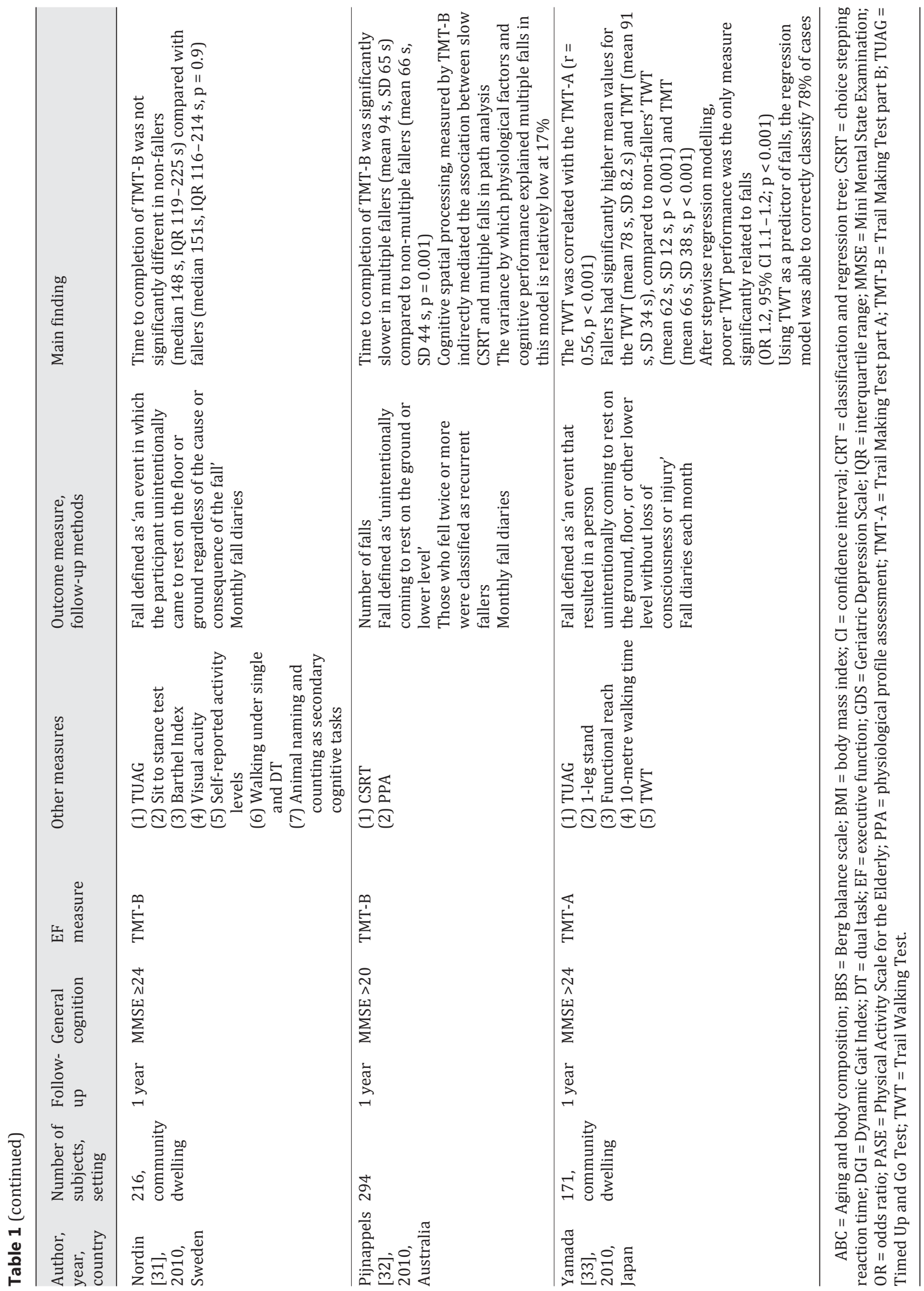


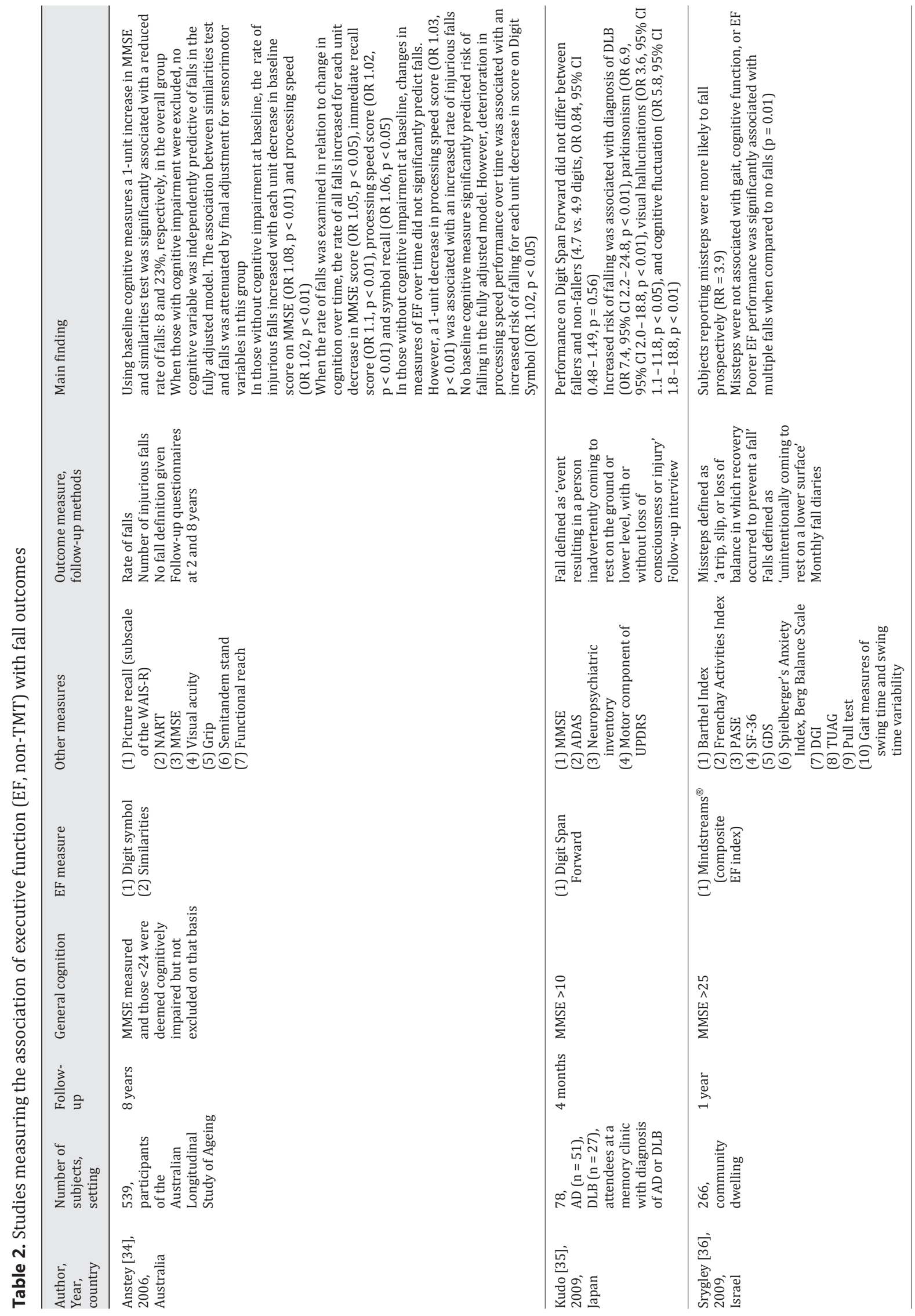


Kearney et al.: The Relationship between Executive Function and Falls and Gait Abnormalities in Older Adults: A Systematic Review

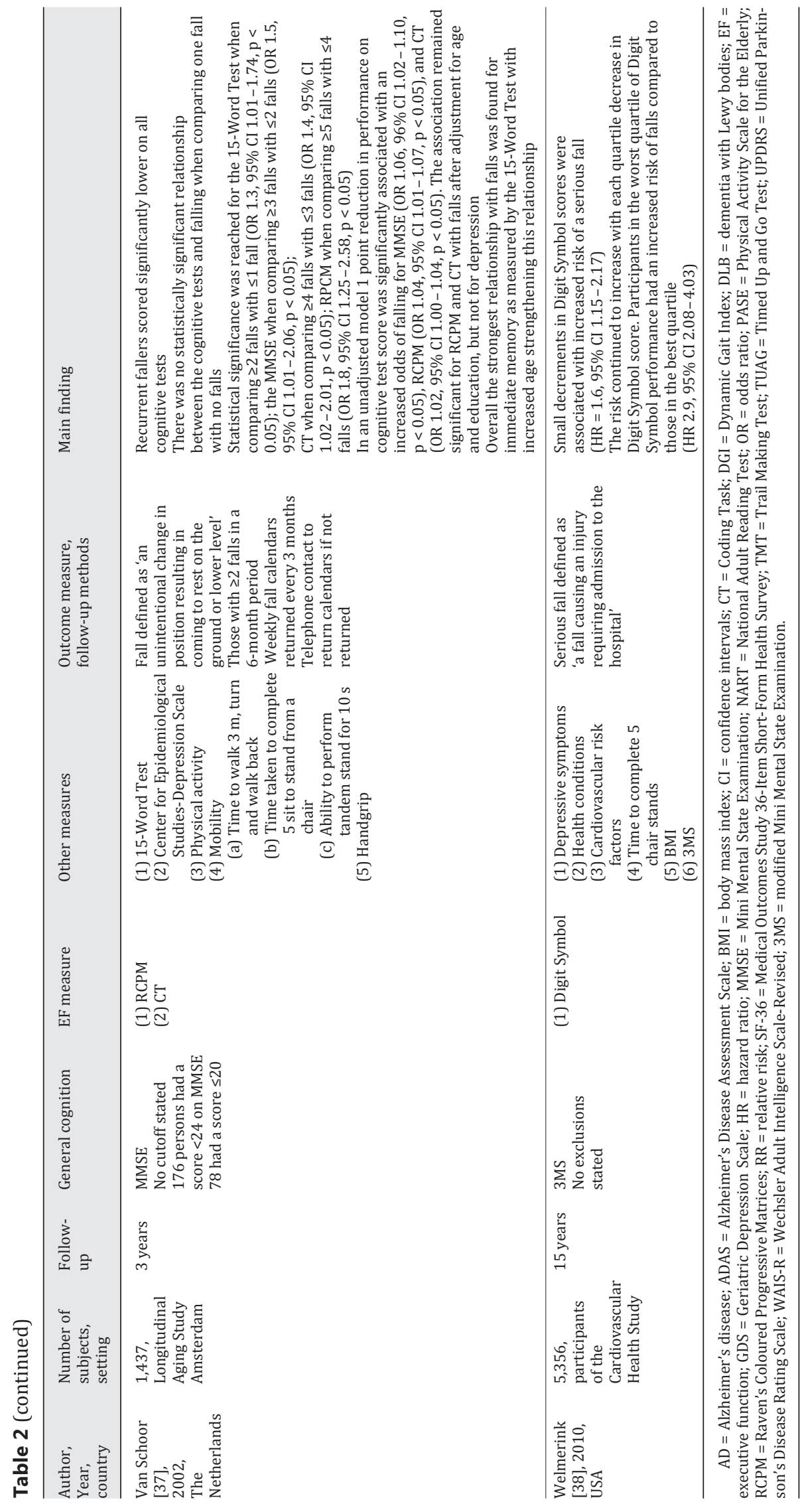


Kearney et al.: The Relationship between Executive Function and Falls and Gait Abnormalities in Older Adults: A Systematic Review

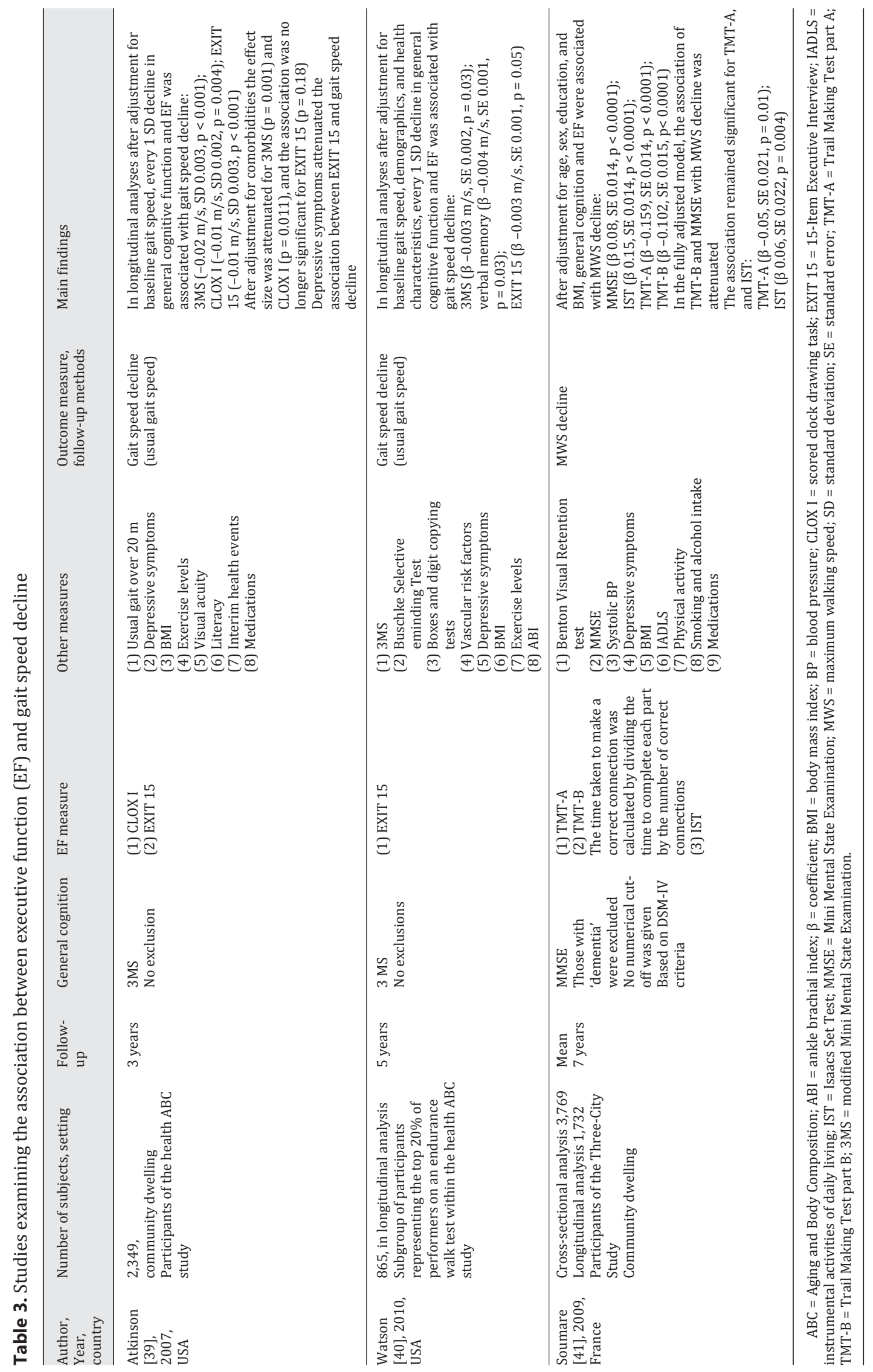


Kearney et al.: The Relationship between Executive Function and Falls and Gait

Abnormalities in Older Adults: A Systematic Review

Table 4. Tests of executive function

\begin{tabular}{|c|c|}
\hline Test name & Description \\
\hline TMT-A & $\begin{array}{l}\text { Participants draw lines to connect consecutively numbered circles; tests how effectively } \\
\text { an individual responds to a visual array and follows a sequence. It also requires motor } \\
\text { speed and agility as it is a test of speed as well as accuracy }\end{array}$ \\
\hline TMT-B & $\begin{array}{l}\text { Participants draw lines to alternatively connect numbered and lettered circles. In } \\
\text { addition to the skills tested by the TMT-A, the TMT-B also requires cognitive flexibility to } \\
\text { deal with more than one visual stimulus and shift between stimuli during the course of an } \\
\text { ongoing activity. The scoring methods for both TMT-A and TMT-B require an examiner to } \\
\text { point out errors as they occur so that the test can be completed without errors and the } \\
\text { scoring is based on time alone }\end{array}$ \\
\hline$\Delta \mathrm{TMT}$ & $\begin{array}{l}\text { This is a score derived from subtracting the time taken to complete the TMT-A from the } \\
\text { time taken to complete the TMT-B and removes the speed element from the test evaluation }\end{array}$ \\
\hline Normalised TMT & TMT (B-A)/A isolates the cognitive flexibility component of the TMT-B \\
\hline $\begin{array}{l}\text { Digit Symbol } \\
\text { Substitution Test } \\
\text { (Digit Symbol) }\end{array}$ & $\begin{array}{l}\text { Consists of rows containing small squares, each paired with a randomly assigned number } \\
\text { from } 1 \text { to } 9 \text {. An accompanying printed key pairs each number with a different nonsense } \\
\text { symbol. Subjects are required to fill the numbered squares with the corresponding } \\
\text { nonsense symbol. The score is the number of correctly filled squares. It is a test of } \\
\text { processing speed }\end{array}$ \\
\hline Coding task & $\begin{array}{l}\text { This task consists of three 1-min trials in which participants have to combine two } \\
\text { characters according to a given example. The score on each trial is the number of } \\
\text { correctly completed combinations irrespective of the number of wrong answers. The } \\
\text { mean score of the three trials is used as the overall score. It is a measure of information- } \\
\text { processing speed }\end{array}$ \\
\hline CLOX I & $\begin{array}{l}\text { Is a scored clock-drawing task whereby the subject is instructed to draw a clock } \\
\text { displaying a particular time on a blank page. It reflects performance in a novel and } \\
\text { ambiguous situation. The participant must choose the clock format (digital, analog face, } \\
\text { watch), size, and position on page. It requires initiation, persistence, sequencing of } \\
\text { constructional actions, progress monitoring, and error correction }\end{array}$ \\
\hline EXIT 15 & $\begin{array}{l}\text { This is a shortened version of the } 25 \text {-Item Executive Interview. The EXIT } 15 \text { assesses } \\
\text { inhibition of automatic responses and intrusions, word and design fluency, and } \\
\text { sequencing tasks }\end{array}$ \\
\hline Mindstreams & $\begin{array}{l}\text { This is a computerised testing system for comprehensive clinical assessment of cognitive } \\
\text { impairment, testing multiple cognitive domains, and designed primarily for use in older } \\
\text { patients. A number of tests may be used to generate and index score for the cognitive } \\
\text { domain of interest such as executive function }\end{array}$ \\
\hline
\end{tabular}

Digit Span Forward Participants are presented with increasing numbers of spoken digits at a rate of $1 / \mathrm{s}$ that have to be repeated in the same order. It begins with 3 digits, and the number of digits presented is increased by 1 in every other trial. When errors in 2 consecutive trials are made, the test is ended. The score is based on the number of correctly recalled trials. This is a test of efficiency of attention

Verbal fluency

Participants have to say as many words as possible from a category in a given time (usually $1 \mathrm{~min}$ ). The category may be semantic such as animals or fruit, or phonemic, such as words beginning with the letter s or $\mathrm{f}$. This test is scored by summing the number of valid words. It is a test of self-regulation

Isaacs Set Test This also tests verbal fluency abilities and speed of verbal production. It measures the ability to generate lists of words in 4 semantic categories (colours, animals, fruit, cities) in $1 \mathrm{~min}$ for each. A total score is summed over the 4 categories

Similarities Participants are asked to explain why two different items are similar, such as 'what have an apple and an orange got in common'. The score is the number of correct associations made. It is a test of verbal abstract reasoning

Raven's Coloured Progressive Matrices
This test consists of 3 sections of 12 items, each showing a visual pattern in which a small part is missing. The participant is asked to choose 1 of 6 possible alternatives that best fits the missing part. It measures non-verbal and abstract reasoning 
Kearney et al.: The Relationship between Executive Function and Falls and Gait Abnormalities in Older Adults: A Systematic Review

A further 5 studies used alternative executive measures at baseline [34-38] (table 2). Three longitudinal studies recorded performance in the Digit Symbol Substitution Test (Digit Symbol) or the Coding task, both measures of processing speed [34, 37, 38]. Participants in the first of these studies also completed a similarity task, a measure of abstract verbal reasoning, which was found to be associated with the rate of falling over an 8-year follow-up [34]. For each additional error in Digit Symbol performance there was a 2\% increased rate of falls (OR 1.02, 95\% CI 1.00-1.03) [34]. Van Schoor et al. [37] also examined immediate memory in addition to abstract reasoning and processing speed. Baseline scores for all cognitive tests were significantly lower in recurrent fallers (defined as $\geq 2$ falls in the same 6-month period). There was no significant relationship between cognitive tests and falls when comparing one fall with no falls. However, the strength of associations of executive function increased in groups defined by progressively increasing the number of falls in the same 6-month period [37]. Additionally, a 1-unit decrease in abstract reasoning and processing speed performance was associated with increased odds of falling, but the significance of the association was attenuated by depressive symptoms. This association was not found with the test of immediate memory [37]. In the largest cohort of 5,356 participants, lower baseline Digit Symbol scores were associated with an increased risk of experiencing a serious fall (hazard ratio, HR, 1.9, 95\% CI 1.4-2.5 for those in the lowest performance quartile compared to the best quartile). Risk of serious fall increased as Digit Symbol performance deteriorated (HR 1.6, 95\% CI 1.2-2.1, for 1-quartile decrease and HR 2.9, 95\% CI 2.1-4.0, for a 3-quartile decrease in Digit Symbol performance over time) [38]. In a study recording both 'missteps' (defined as 'a trip, slip, or loss of balance in which recovery occurred to prevent a fall') and falls as outcome measures, a composite measure of executive function (Mindstreams) was significantly associated with multiple falls, while no similar association was found with missteps [36]. In a small group of subjects $(n=78)$ with either Alzheimer's disease or dementia with Lewy bodies, Digit Span Forward was not predictive of future falls [35].

The remaining 3 studies evaluated both general cognition and executive function and their association with gait speed decline in longitudinal analyses [39-41]. Two studies analysed and reported a different data set from the Health, Aging and Body Composition (Health ABC) study $[39,40]$. Associations were found between executive function and gait speed in cross-section and with gait speed decline in longitudinal analyses in all 3 studies. A scored clock drawing task was predictive of gait speed decline in the original Health $\mathrm{ABC}$ cohort, while the association with a more comprehensive executive assessment, the Exit Interview (Exit 15), was attenuated by depressive symptoms [39]. However, results from the later Health $\mathrm{ABC}$ cohort found an association between Exit 15 and gait speed decline in a subgroup with better physical performance at baseline [40]. Soumare et al. [41] also found a significant association between TMT-A and verbal fluency and gait speed decline.

No studies examining an association between executive function and balance were identified by this review.

\section{Discussion}

This study presents a systematic review of cohort studies from 1991 to April 2011 examining the association between measures of executive function and future fall risk, or changes in gait parameters in older adults. Nine of the 11 studies recording falls as the primary outcome found an association with a baseline measure of executive function. All 3 studies examining gait speed found associations between processing speed and gait speed decline in longitudinal analyses [39-41]. 
The Trail Making Test was the most commonly used measure of executive function and poor baseline performance was associated with future falls. The majority of included studies analysed recurrent/multiple falls or serious/injurious falls rather than single falls. This may indicate that impaired executive function is associated with a more clinically relevant falling pattern or it may reflect a more functionally dependent cohort, albeit still community dwelling, and an association with increasing frailty. The latter is in keeping with results from a study of Medicare patients in the USA which reported an association between recurrent falls and increased age, fair or poor health, and limitations in activities in daily living [42]. A possible association of executive function with frailty has also been identified by another research group [43].

Two studies suggest that executive function is particularly relevant in older adults who have already declined physiologically [30, 32]. In a study recording 'missteps' and falls, missteps predicted falls suggesting associated balance impairment. However, missteps may be adaptive to impaired physiology and occurred to prevent falls in those with balance impairment. Participants reporting missteps had better executive function, potentially reflecting better judgement and reaction times resulting in avoidance of some falls by taking a protective misstep. In which case, as executive function declined, the adaptive strategy failed resulting in falls rather than missteps, potentially explaining the association between impaired executive function and recurrent falls but not missteps [36].

Results from 3 longitudinal ageing studies indicate that deterioration in cognition may be more relevant to fall risk than single measurements [34, 37, 38]. In these studies, as cognitive performance declined, risk offalls increased. Also the degree of executive dysfunction seems more important than a simple dichotomous presence or absence of impairment. In two studies, each using different executive function measures, those in the worst performance quartile of each test were approximately 3 times more likely to fall compared to those in the best quartile [17, 38], suggesting a graded association between executive function and fall risk. Determining fall risk on the basis of cognitive change and extent of impairment may be more relevant clinically as most cognitive decline is progressive. Those individuals who experience a more rapid decline or have more severe impairment may have less potential to adapt to their deficits thus conferring a higher fall risk.

Whether executive function influences gait through judgement, reaction time and psychomotor control or whether the association merely reflects a shared anatomical substrate remains unclear. This review indicates that impaired executive function is associated with gait speed decline, but gait speed decline has also been shown to predict emerging cognitive impairment [44]. Subcortical ischaemic vascular disease has been associated with poorer performance in tests of multiple cognitive domains, including executive function, attention, and working memory [45] and has also been associated with falls [46] and gait speed decline [47] in a general older population suggesting that prevalent ischaemic cerebrovascular disease may contribute to the association between executive function, cognitive impairment and falls. Large-scale epidemiological studies further examining this association are required.

The TMT is a widely used test of scanning and visuomotor tracking, divided attention, and cognitive flexibility [12]. It tests speed as well as accuracy. Some studies simply reported TMT-B scores alone, while others corrected for time taken to complete part A, isolating the contribution of cognitive flexibility. Digit Symbol and Coding are tests of psychomotor performance with motor persistence, sustained attention, response speed, and visuomotor coordination playing important roles in their completion [12]. Drawing from theoretical models in neuropsychology, executive function can be divided into the ability to form, maintain and shift mental set [13]. These components correspond to abilities to reason and solve problems, maintain motivation to follow through with a response, and alter goals in response to changing contingencies. In the context of locomotion, this skill set could influence a subject's ability 
Kearney et al.: The Relationship between Executive Function and Falls and Gait Abnormalities in Older Adults: A Systematic Review

to make sound judgement with regard to gait, initiate movement, prioritise walking over secondary tasks to minimise risk, maintain motivation to reach an end point, concentrate and adapt to challenges. Failure in any component of this pathway has potential to increase fall risk. As single tests, TMT and Digit Symbol examine a number of these key components, but describing gait and fall risk in terms of deficits in components of executive function rather than a deficiency on single tests will be more informative. This will allow greater scope to identify deficits in skills related to locomotion and potentially identify targets for training or fall prevention.

The association between impaired ability to 'dual-task' (simultaneously perform a secondary cognitive task while walking without significant impairment of either task) was not examined in this review. This association has been comprehensively reviewed with demonstration of a fivefold increased risk of falls in association with impaired capacity to carry out dual tasks [11]. Dual-task impairment is thought to clinically represent the inability to appropriately divide attention between two cognitively demanding tasks. Individuals who fail to adopt a 'posture-first' strategy and compromise the attention paid to gait are at an increased risk of falls [48]. This may represent poor judgement in failing to prioritise posture or it may fit more closely with lack of cognitive flexibility to adapt and prioritise gait in circumstances of challenge, such as obstacles, or changes in surface and lighting. Therefore, taking the results of this review and those findings from previous dual-task studies, cognitive flexibility to adapt to changing contingencies seems to be a key element of executive function that is associated with increased risk of falls. In older adults processing speed may reflect the ability to 'react' to challenges in walking and may explain the association between impaired processing speed and fall risk found in this review. It is impossible to definitively separate out all components of executive function. Failure in any component has the potential to lead to an increased risk of falls. However, there are components of executive function that seem to predominate in the studies in this review. It may be possible to lack judgement with regard to walking, but if the abilities to 'react and adapt' are preserved, then safe passage may occur even if the activity was ill-advised in the first place. Equally good judgement may not always compensate for inability to adapt to challenges. In the absence of being able to truly isolate all components of executive function, emphasis on assessment of cognitive flexibility and processing speed may hold most clinical relevance when assessing fall risk.

Gait speed decline is associated with falls, but measures of gait variability may be more discriminating. Increased variability of stride length and swing time have been shown to best predict falls, and to be the only predictors of injurious falls, in an older population [18]. Increased stride length variability has been observed in subjects with Alzheimer's disease compared to controls [49]. Executive function has been associated with measures of gait variability in cross-section, but longitudinal analyses examining executive function as a predictor of gait variability and a potential mediator of high fall rates in people with dementia have not been completed.

The strengths of this study are its rigorous design, adherence to a systematic protocol, and independent reviewers contributing to selection of included studies. It is limited by lack of consensus regarding definition of executive function and lack of uniformity in testing measures. This may have excluded studies intending to examine the proposed associations, but that failed to meet the inclusion criteria. No studies reporting balance outcomes were identified by the search. Meta-analyses of findings were not feasible due to lack of uniformity in testing measures and outcomes reported.

This review systematically examined the role of executive function in the longitudinal prediction of falls or gait decline. Impaired executive function is associated with both falls and gait speed decline in older adults, but impaired executive function may be particularly relevant in frail older adults with balance and gait impairments, who rely on cognitive abilities to 
Kearney et al.: The Relationship between Executive Function and Falls and Gait Abnormalities in Older Adults: A Systematic Review

compensate for physical deficits. This review highlights the relationship between executive function (cognitive flexibility and processing speed in particular), falls, and gait decline among a relatively cognitively preserved population. However, since declining executive function is associated with increasing risk, these factors may play an even greater role in those with cognitive impairment and dementia as impaired executive function is likely to be more pervasive in these conditions [50]. This review also identifies a target population in whom fall prevention should be optimised. Research studies aiming to improve executive function through exercise, dual-task training, or cognitive enhancing medications have shown promising results [51]. The potential to train gait abnormalities has also been demonstrated in a Parkinson's disease population [52] and warrants evaluation in subjects with cognitive impairment. Those with mild cognitive impairment may represent a group in whom executive deficits and gait abnormalities, if identified, could be trained or modified before the extent of cognitive decline means fall prevention strategies are adaptive and restrictive rather than rehabilitative. Novel approaches adapting rehabilitation strategies to the needs of an individual with executive dysfunction and cognitive impairment and evaluation of such interventions in randomised controlled trials are now required.

\section{Acknowledgements}

This research was funded by a research grant awarded by the Nottinghamshire County NHS Trust.

\section{Disclosure Statement}

Competing interests: none.

\section{References}

1 Tinetti ME, Speechley M, Ginter SF: Risk factors for falls among elderly persons living in the community. N Engl J Med 1988;319:1701-1707.

2 Shaw FE: Falls in cognitive impairment and dementia. Clin Geriatr Med 2002;18:159-173.

- 3 Van Dijk PT, Meulenberg OG, van de Sande HJ, Habbema JD: Falls in dementia patients. Gerontologist 1993;33: 200-204.

4 Kallin K, Gustafson Y, Sandman P-0, Karlsson S: Factors associated with falls among older, cognitively impaired people in geriatric care settings: a population-based study. Am J Geriatr Psychiatry 2005;13:501-509.

- 5 Tinetti ME, Allore H, Araujo KLB, Seeman T: Modifiable impairments predict progressive disability among older persons. J Aging Health 2005;17:239-256.

6 Panel on Prevention of Falls in Older Persons American Geriatrics Society, British Geriatrics Society: Summary of the Updated American Geriatrics Society/British Geriatrics Society clinical practice guideline for prevention of falls in older persons. J Am Geriatr Soc 2011;59:148-157.

- 7 Oliver D, Connelly JB, Victor CR, et al: Strategies to prevent falls and fractures in hospitals and care homes and effect of cognitive impairment: systematic review and meta-analyses. BMJ 2007;334:82.

- 8 Shaw FE, Bond J, Richardson DA, et al: Multifactorial intervention after a fall in older people with cognitive impairment and dementia presenting to the accident and emergency department: randomised controlled trial. BMJ 2003;326:73, erratum in BMJ 2003;326:699.

- 9 Shaw FE: Prevention of falls in older people with dementia. J Neural Transm 2007;114:1259-1264.

10 Lundin-Olsson L, Nyberg L, Gustafson Y: 'Stops walking when talking' as a predictor of falls in elderly people. Lancet 1997;349:617.

11 Beauchet 0 , Annweiler C, Dubost V, et al: Stops walking when talking: a predictor of falls in older adults? Eur J Neurol 2009;16:786-795.

12 Lezak MD, Howieson DB, Loring DW: Neurospychological Assessment, ed 4. New York, Oxford University Press, 2004.

13 Suchy Y: Executive functioning: overview, assessment, and research issues for non-neuropsychologists. Ann Behav Med 2009;37:106-116.

14 Nevitt MC, Cummings SR, Hudes ES: Risk factors for injurious falls: a prospective study. J Gerontol 1991; 46:M164-M170. 
Kearney et al.: The Relationship between Executive Function and Falls and Gait

Abnormalities in Older Adults: A Systematic Review

15 Rapport LJ, Hanks RA, Millis SR, Deshpande SA: Executive functioning and predictors of falls in the rehabilitation setting. Arch Phys Med Rehabil 1998;79:629-633.

16 Sheridan PL, Hausdorff JM: The role of higher-level cognitive function in gait: executive dysfunction contributes to fall risk in Alzheimer's disease. Dementia Geriatr Cogn Disord 2007;24:125-137.

17 Herman T, Mirelman A, Giladi N, Schweiger A, Hausdorff JM: Executive control deficits as a prodrome to falls in healthy older adults: a prospective study linking thinking, walking, and falling. J Gerontol Ser A Biol Sci Med Sci 2010;65:1086-1092.

18 Verghese J, Holtzer R, Lipton RB, Wang C: Quantitative gait markers and incident fall risk in older adults. J Gerontol Ser A Biol Sci Med Sci 2009;64:896-901.

19 Merory JR, Wittwer JE, Rowe CC, Webster KE: Quantitative gait analysis in patients with dementia with Lewy bodies and Alzheimer's disease. Gait Post 2007;26:414-419.

20 Nadkarni NK, Mawji E, Mcllroy WE, Black SE: Spatial and temporal gait parameters in Alzheimer's disease and aging. Gait Post 2009;30:452-454.

-21 Allan LM, Ballard CG, Burn DJ, Kenny RA: Prevalence and severity of gait disorders in Alzheimer's and nonAlzheimer's dementias. J Am Geriatr Soc 2005;53:1681-1687.

-22 Allali G, Assal F, Kressig RW, Dubost V, Herrmann FR, Beauchet O: Impact of impaired executive function on gait stability. Dementia Geriatr Cogn Disord 2008;26:364-369.

-23 Yogev-Seligmann G, Hausdorff JM, Giladi N: The role of executive function and attention in gait. Mov Disord 2008;23:329-342, quiz 472.

24 Ble A, Volpato S, Zuliani G, et al: Executive function correlates with walking speed in older persons: the InCHIANTI study. J Am Geriatr Soc 2005;53:410-415.

25 Holtzer R, Verghese J, Xue X, Lipton RB: Cognitive processes related to gait velocity: results from the Einstein Aging Study. Neuropsychology 2006;20:215-223.

-26 Hausdorff JM, Yogev G, Springer S, Simon ES, Giladi N: Walking is more like catching than tapping: gait in the elderly as a complex cognitive task. Exp Brain Res 2005;164:541-548.

27 Stroup DF, Berlin JA, Morton SC, et al: Meta-analysis of observational studies in epidemiology: a proposal for reporting. Meta-Analysis of Observational Studies in Epidemiology (MOOSE) group. JAMA 2000;283:20082012.

28 Moher D, Liberati A, Tetzlaff J, Altman DG, Group P: Preferred reporting items for systematic reviews and meta-analyses: the PRISMA statement. BMJ 2009;339:b2535.

29 The Newcastle-Ottawa quality assessment scale. 2009. http://www.ohri.ca/programs/clinical_epidemiology/oxford.asp (accessed October 14, 2011).

30 Delbaere K, Close JCT, Heim J, et al: A multifactorial approach to understanding fall risk in older people. J Am Geriatr Soc 2010;58:1679-85.

-31 Nordin E, Moe-Nilssen R, Ramnemark A, Lundin-Olsson L: Changes in step-width during dual-task walking predicts falls. Gait Post 2010;32:92-97.

-32 Pijnappels M, Delbaere K, Sturnieks DL, Lord SR: The association between choice stepping reaction time and falls in older adults - a path analysis model. Age Ageing 2010;39:99-104.

-33 Yamada M, Ichihashi N: Predicting the probability of falls in community-dwelling elderly individuals using the trail-walking test. Environmental Health and Preventive Medicine 2010;15:386-391.

-34 Anstey KJ, von Sanden C, Luszcz MA: An 8-year prospective study of the relationship between cognitive performance and falling in very old adults. J Am Geriatr Soc 2006;54:1169-1176.

-35 Kudo Y, Imamura T, Sato A, Endo N: Risk factors for falls in community-dwelling patients with Alzheimer's disease and dementia with Lewy bodies: walking with visuocognitive impairment may cause a fall. Dementia Geriatr Cogn Disord 2009;27:139-146.

-36 Srygley JM, Herman T, Giladi N, Hausdorff JM: Self-report of missteps in older adults: a valid proxy of fall risk? Arch Phys Med Rehabil 2009;90:786-792.

-37 Van Schoor NM, Smit JH, Pluijm SMF, Jonker C, Lips P: Different cognitive functions in relation to falls among older persons. Immediate memory as an independent risk factor for falls. J Clin Epidemiol 2002;55:855-862.

-38 Welmerink DB, Longstreth WT Jr, Lyles MF, Fitzpatrick AL: Cognition and the risk of hospitalization for serious falls in the elderly: results from the Cardiovascular Health Study. J Gerontol Ser A Biol Sci Med Sci 2010;65: 1242-1249.

-39 Atkinson HH, Rosano C, Simonsick EM, et al: Cognitive function, gait speed decline, and comorbidities: the Health, Aging and Body Composition study. J Gerontol Ser A Biol Sci Med Sci 2007;62A:844-850.

$\checkmark 40$ Watson NL, Rosano C, Boudreau RM, et al: Executive function, memory, and gait speed decline in well-functioning older adults. J Gerontol Ser A Biol Sci Med Sci 2010;65:1093-1100.

-41 Soumare A, Tavernier B, Alperovitch A, Tzourio C, Elbaz A: A cross-sectional and longitudinal study of the relationship between walking speed and cognitive function in community-dwelling elderly people. J Gerontol Ser A Biol Sci Med Sci 2009;64:1058-1065.

42 Shumway-Cook A, Ciol MA, Hoffman J, Dudgeon BJ, Yorkston K, Chan L: Falls in the Medicare population: incidence, associated factors, and impact on health care. Phys Ther 2009;89:324-332.

43 Morley JE: Developing novel therapeutic approaches to frailty. Curr Pharm Des 2009;15:3384-3395.

$\checkmark 44$ Marquis S, Moore MM, Howieson DB, et al: Independent predictors of cognitive decline in healthy elderly persons. Arch Neurol 2002;59:601-606. 
45 Jokinen H, Kalska H, Ylikoski R, et al: Longitudinal cognitive decline in subcortical ischemic vascular disease - the LADIS Study. Cerebrovasc Dis 2009;27:384-391.

46 Srikanth V, Beare R, Blizzard L, et al: Cerebral white matter lesions, gait, and the risk of incident falls: a prospective population-based study. Stroke 2009;40:175-180.

47 Rosano C, Kuller LH, Chung H, Arnold AM, Longstreth WT Jr, Newman AB: Subclinical brain magnetic resonance imaging abnormalities predict physical functional decline in high-functioning older adults. J Am Geriatr Soc 2005;53:649-654.

48 Beauchet O, Dubost V, Allali G, Gonthier R, Hermann FR, Kressig RW: 'Faster counting while walking' as a predictor of falls in older adults. Age Ageing 2007;36:418-423.

-49 Webster KE, Merory JR, Wittwer JE: Gait variability in community dwelling adults with Alzheimer disease. Alzheimer Dis Assoc Disord 2006;20:37-40.

50 Voss SE, Bullock RA: Executive function: the core feature of dementia? Dementia Geriatr Cogn Disord 2004; 18:207-216.

-51 Segev-Jacubovski 0, Herman T, Yogev-Seligmann G, Mirelman A, Giladi N, Hausdorff JM: The interplay between gait, falls and cognition: can cognitive therapy reduce fall risk? Expert Rev 2011;11:1057-1075.

52 Frenkel-Toledo S, Giladi N, Peretz C, Herman T, Gruendlinger L, Hausdorff JM: Treadmill walking as an external pacemaker to improve gait rhythm and stability in Parkinson's disease. Mov Disord 2005;20:1109-1114. 